\title{
Blood Stream Infections in Intensive Care Units and its Antibiogram
}

\section{Mariyah Yousuf $^{1 *}$, Tarana Sarwat ${ }^{2}$, Sneha K Mohan², Nikita Birhma ${ }^{1}$ and Dalip K Kakru ${ }^{3}$}

${ }^{1}$ Msc Medical Microbiology, Department of Microbiology, School of Medical Sciences and Research, Sharda University, Greater Noida, UP, India

${ }^{2}$ Assistant Professor, Department of Microbiology School of Medical Sciences and Research, Sharda University, Greater Noida, UP, India

${ }^{3}$ Professor, Department of Microbiology, School of Medical Sciences and Research, Sharda University, Greater Noida, UP, India

*Corresponding Author: Mariyah Yousuf, Msc Medical Microbiology, Department of Microbiology, School of Medical Sciences and Research, Sharda University, Greater Noida, UP, India.

\author{
Received: May 01, 2020 \\ Published: May 28, 2020 \\ (C) All rights are reserved by Mariyah \\ Yousuf., et al.
}

\begin{abstract}
Introduction: Early diagnosis and appropriate treatment of blood stream infections can make the difference between life and death. It would reduce mortality from septicaemia, reduce turn around time and improve patient management. This study aims at the microbiological characteristics and antibiotic susceptibility of BSI in intensive care units.

Materials and Methods: A total of 670 blood samples received in the bacteriology laboratory 112 samples showed culture positivity. Results: Coagulase negative Staphylococcus, represent the most commonly isolated organisms. Among the gram negative organisms Pseudomonas species, Escherichia coli, Klebsiella and Enterococcus species were commonly isolated.

Conclusion: Bacteriological etiology of sepsis along with the antibiogram of septicemic isolates provide necessary information for the formulation of antibiotic policy in effective management of such cases in intensive care units.

Keywords: BSI; Antimicrobial Susceptibility; Bacteremia; ICU
\end{abstract}

\section{Abbreviations}

BSI: Blood Stream Infections; Spp: Species; ICU: Intensive Care Unit; CLSI: Clinical and Laboratory Standards Institute Guidelines; MICU: Medical Intensive Care Unit; SICU: Surgical Intensive Care Unit; PICU: Paediatric Intensive Care Unit; NICU: Neonatal Intensive Care Unit; CONS: Coagulase Negative Staphylococcus; GPC: Gram Positive Cocci

\section{Introduction}

Bacteremia is related to the presence of viable bacteria in the blood confirmed by cultures in which contamination has been effectively ruled out [1,2]. Critically ill patients are particularly predisposed to the acquisition of BSIs, which occur in approximately $7 \%$ of all patients within the first month of hospitalization in Intensive Care Unit (ICU) [3]. The Extended Prevalence of Infection in the ICU Study (EPIC II) conducted in 2007 showed that in ICU approximately $15 \%$ of patients had a BSI on the day of the study [4]. The extensive use of intravascular catheters is recognized as the most important factor contributing to the occurrence of BSI $[5,6]$.
Central venous catheters, in particular, represent the intravascular devices that are most frequently associated with the acquisition of a BSI, although arterial catheters can be also involved [7-9].

Catheter-related BSIs (CR-BSIs) (defined as the growth of the same pathogen from catheter tip and peripheral blood culture), which represent up to $30 \%$ of cases, and primary BSIs, accounting for around $35 \%$ of cases, are the most common types of BSI in ICU $[10,11]$. Ventilator-Associated-Pneumonia (VAP), which is a frequent complication when mechanical ventilation is required, is bacteremic in around 15\% of cases, and represent the most common source of secondary bacteremia in critically ill patients $[11,12]$. The most common bacteria that cause bacteremia include members of Staphylococcus, Streptococcus, Enterococcus, Escherichia coli, Klebsiella, Pseudomonas, Enterobacter, Haemophilus and Neisseria genera [13-15]. Blood culture remains the highly specific indicator of bacteremia and antimicrobial susceptibility test assist a great deal in precise identification of the most appropriate choice of drug to be administer. 


\section{Materials and Methods}

The study was conducted in the Department of Microbiology, Central Laboratory, School of Medical Sciences and Research, Sharda Hospital, Greater Noida. It is a prospective study which was conducted for a period of 6 months. All the isolates from blood culture positive samples collected over period of six months were assessed. In this study we adopted automated method i.e. (BACTALERT system) in order to culture the bottles received in the bacteriology laboratory. The bottles were put inside the automated machine, when there was a signal the bottles were removed and the blood from the positive blood culture bottles was subjected to subculture on 5\% sheep blood agar and MacConkey agar. The growth on culture plates was identified on the basis of colony morphology, Gram stain, and various biochemical tests. Antimicrobial susceptibility testing were performed for all blood cultures isolated on Muller Hinton agar by Kirby- Bauer disc diffusion method as recommended in the CLSI (Clinical And Laboratory Standards Institute guidelines) 2019. Commercially available antibiotics disks (Hi media) were used for antimicrobial susceptibility testing. A total of 670 blood samples were received in the bacteriology lab for culture out of which 112 samples showed culture positivity. All the isolates were identified on the basis of culture characteristics, Gram stain and biochemical reactions. The isolates were then subjected to antimicrobial susceptibility testing.

\section{Results}

A total of 670 blood samples were received in the bacteriology lab for culture out of which 112 samples showed culture positivity. All the isolates were identified on the basis of culture characteristics, Gram stain and biochemical reactions. The isolates were then subjected to antimicrobial susceptibility testing. Out of 112 positive samples obtained, 74 were male patients and 38 were females indicating higher rates of blood stream infections in males as compared to females. The maximum number of samples was obtained from patients between age 60 - 70 years in MICU (Table 1 and figure 1). Maximum number of cases were obtained from medical ICU approximately $46 \%$ followed by surgical ICU approximately $26 \%$. Gram positive staphylococci constituted maximum proportion of isolates followed by Pseudomonas species and E. coli. (Table 8 and figure 8). Amongst all the antibiotics, Linezolid (100\%) and vancomycin (100\%) emerged as most sensitive drug against Staphylococcus aureus. Strains of Staphylococcus aureus showed high resistance for erythromycin (75\%) and cefoxitin (62\%). Amongst all the antibiotics, linezolid (83\%). Gentamicin (66\%), Levofloxacin (66\%), vancomycin (66\%), ciprofloxacin (66\%) emerged as most sensitive drug against Enterococcus spp (Table 4 and figure 6). Strains of Enterococcus spp showed high resistance for ampicillin
$(83 \%)$ followed by penicillin (66\%). Gentamycin (94\%) emerged as most sensitive drug against $E$. coli (Table 5 and figure 7) followed by meropenem (92\%), and cotrimoxazole (82\%). Strains of Escherichia coli showed high resistance for cefuroxime (65\%) followed by ceftriaxone (65\%) and ampicillin (53\%). Imipenem (89\%) meropenem $(77 \%)$ emerged as most sensitive drug against Klebsiella followed by cotrimoxazole (67\%). Strains of Klebsiella (Table 6 and figure 8) showed high resistance for cefepime (89\%) followed by ceftriaxone (78\%) and Ampicillin (78\%). Gentamicin (87\%) emerged as most sensitive drug against Citrobacter followed by imipenem (75\%) and cotrimoxazole (75\%). Strains of Citrobacter showed high resistance for cefepime $(75 \%)$ followed by ampicillin (62\%). Amongst all the antibiotics, Piperacillin + Tazobactam (87\%) emerged as most sensitive drug against Pseudomonas followed by piperacillin (75\%) and ciprofloxacin (75\%). Strains of Pseudomonas (Table 7 and figure 9) showed high resistance for Aztreonam (63\%) followed by ceftazidime (51\%) and tobramycin (33\%). Gentamicin (71\%), Levofloxacin (71\%) emerged as most sensitive drug against Acinetobacter followed by piperacillin - tazobactam (57\%) and cotrimoxazole (42\%). Strains of Acinetobacter spp showed high resistance for tetracycline (85\%) followed by ceftriaxone (71\%).

\begin{tabular}{|l|c|c|c|}
\hline \multicolumn{1}{|c|}{ ICU } & Total cases & Males & Females \\
\hline Medical ICU & $52(48 \%)$ & 36 & 16 \\
\hline Surgical ICU & $26(23 \%)$ & 17 & 9 \\
\hline Pead. ICU & $23(20.5 \%)$ & 13 & 10 \\
\hline Neonatal ICU & $11(10 \%)$ & 8 & 3 \\
\hline
\end{tabular}

Table 1: Department wise distribution of samples.



Figure 1: Department wise distribution of samples. 


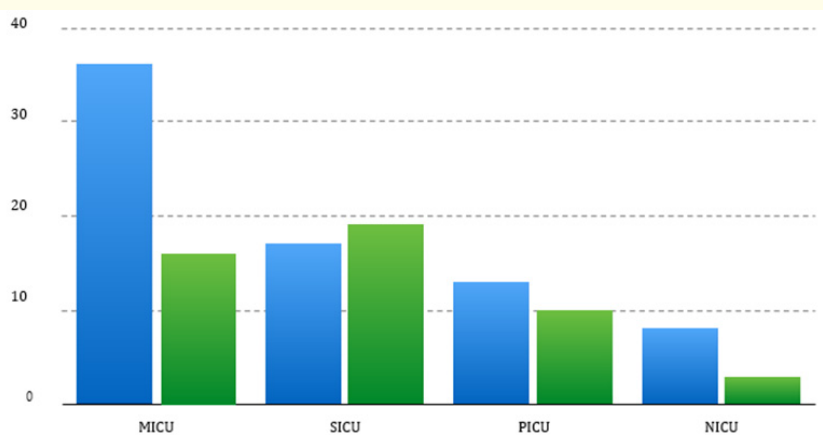

Figure 2: Number of positive cases based on gender in various ICUs.

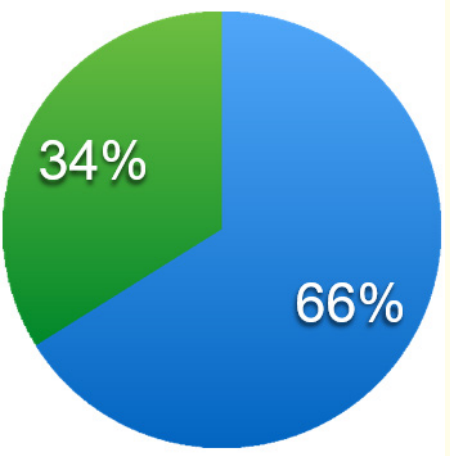

Figure 3: Male to female ratio of positive case in our study.

\begin{tabular}{|l|c|c|}
\hline \multicolumn{1}{|c|}{ Organism } & Number & Percentage \\
\hline Staphylococcus aureus & 8 & 7.1 \\
\hline Enterococcus & 8 & 7.1 \\
\hline E. coli & 17 & 15.2 \\
\hline Klebsiella spp & 8 & 7.1 \\
\hline Citrobacter spp & 7 & 6.2 \\
\hline Pseudomonas spp & 22 & 19.6 \\
\hline Acinetobacter spp & 7 & 6.2 \\
\hline CONS & 35 & 31.2 \\
\hline
\end{tabular}

Table 2: Distribution of organisms in positive samples.

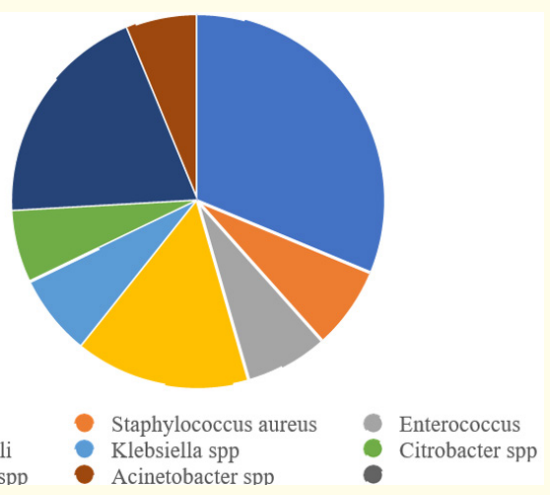

Escherichia coli

Pseudomonas sp

Acinetobacter sp

Citrobacter spp

Figure 4: Distribution of organisms in positive samples.

\begin{tabular}{|l|c|c|}
\hline \multicolumn{1}{|c|}{ Antibiotics } & Sensitive \% & Resistant \% \\
\hline Vancomycin & 100 & 0 \\
\hline Linezolid & 90 & 9.3 \\
\hline Gentamicin & 78 & 23 \\
\hline Ciprofloxacin & 40 & 59 \\
\hline Levofloxacin & 54 & 54 \\
\hline Erythromycin & 23 & 76 \\
\hline Penicillin & 34 & 65 \\
\hline Clindamycin & 55 & 44 \\
\hline Cefotaxime & 46 & 53 \\
\hline
\end{tabular}

Table 3: Antibiotic susceptibility pattern of coagulase negative Staphylococcus (CoNS).

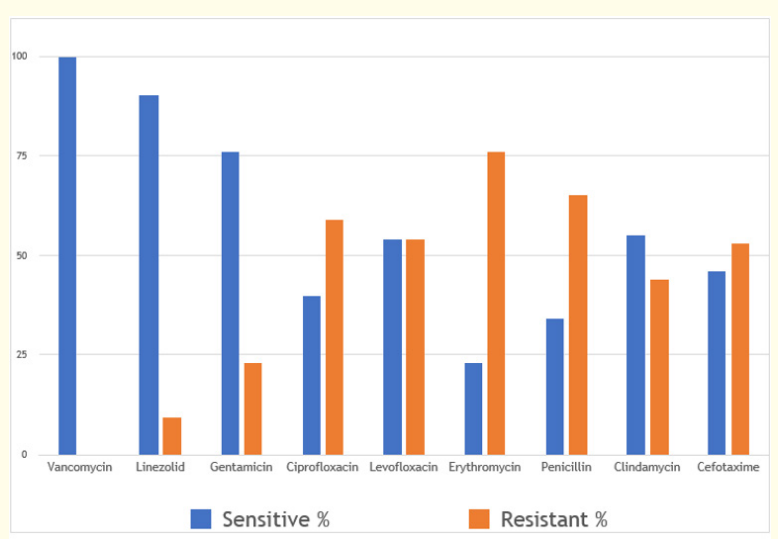

Figure 5: Antibiotic susceptibility pattern of CoNS.

\begin{tabular}{|c|c|c|}
\hline Antibiotics & Sensitive n \% & Resistant n \% \\
\hline Ampicillin & $01(16 \%)$ & $05(83 \%)$ \\
\hline Ciprofloxacin & $04(66 \%)$ & $02(33 \%)$ \\
\hline Gentamicin & $04(66 \%)$ & $02(33 \%)$ \\
\hline Levofloxacin & $04(66 \%)$ & $02(33 \%)$ \\
\hline Linezolid & $05(83 \%)$ & $01(16 \%)$ \\
\hline Penicillin & $02(33 \%)$ & $04(66 \%)$ \\
\hline Streptomycin & $03(50 \%)$ & $03(50 \%)$ \\
\hline Tetracycline & $03(50 \%)$ & $03(50 \%)$ \\
\hline Vancomycin & $04(66 \%)$ & $02(33 \%)$ \\
\hline Teicoplanin & $04(66 \%)$ & $02(33 \%)$ \\
\hline
\end{tabular}

Table 4: Antibiotic susceptibility pattern of Enterococcus spp.

\section{Discussion}

In this study among total microorganisms causing BSI, 54\% were Gram-negative and $45 \%$ were Gram-positive which correlates with study of Vincenzo Russotto, Andrea Cortegiani., et al. [16], in which total microorganisms causing BSI, 57.6\% were 


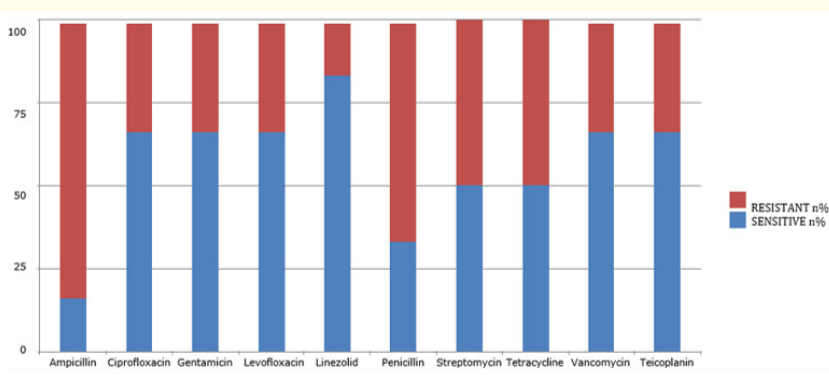

Figure 6: Antibiotic susceptibility pattern of Enterococcus spp.

\begin{tabular}{|l|c|c|}
\hline \multicolumn{1}{|c|}{ Antibiotics } & Sensitive n \% & Resistant n \% \\
\hline Ampicillin & $08(47 \%)$ & $09(53)$ \\
\hline Amoxyclav & $09(53 \%)$ & $8(47 \%)$ \\
\hline Cefepine & $13(76 \%)$ & $4(24 \%)$ \\
\hline Cefotaxime & $08(47 \%)$ & $9(53 \%)$ \\
\hline Ceftriaxone & $06(35 \%)$ & $11(65 \%)$ \\
\hline Cefuroxime & $06(35 \%)$ & $11(65 \%)$ \\
\hline Gentamicin & $16(94 \%)$ & $1(6 \%)$ \\
\hline Imipenem & $14(82 \%)$ & $3(18 \%)$ \\
\hline Levofloxacin & $11(65 \%)$ & $6(35 \%)$ \\
\hline Meropenem & $13(92 \%)$ & $4(8 \%)$ \\
\hline Cotrimoxazole & $14(82 \%)$ & $3(18 \%)$ \\
\hline
\end{tabular}

Table 5: Antibiotic susceptibility pattern of Escherichia coli.

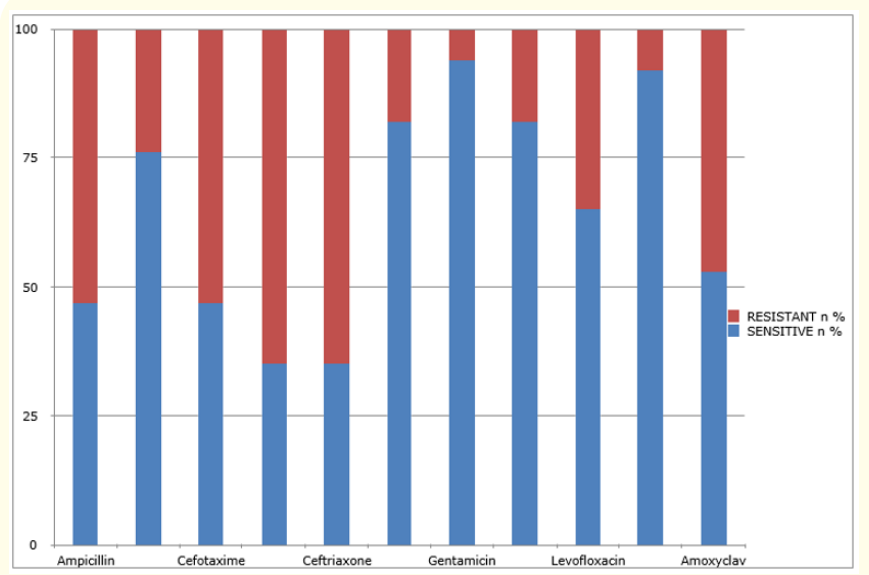

Figure 7: Antibiotic susceptibility pattern of Enterococcus spp.

Gram-negative and 33.4\% were Gram-positive. Gram positive bacteria accounted for $46 \%$ of the isolates. Among gram positive bacteria Coagulase negative Staphylococcus (CONS) was isolated in most samples (69\%) than Staphylococcus aureus (15.6\%) which is consistent with study of David K. Warren, Jeanne E Zack., et al.

\begin{tabular}{|l|c|c|}
\hline \multicolumn{1}{|c|}{ Antibiotics } & Sensitive (n \%) & Resistant (n \%) \\
\hline Ampicillin & $02(22 \%)$ & $07(78 \%)$ \\
\hline Amoxyclav & $04(45 \%)$ & $05(55 \%)$ \\
\hline Cefepime & $01(11 \%)$ & $08(89 \%)$ \\
\hline Cefotaxime & $03(33 \%)$ & $06(67 \%)$ \\
\hline Ceftriaxone & $02(22 \%)$ & $07(78 \%)$ \\
\hline Cefuroxime & $04(45 \%)$ & $05(55 \%)$ \\
\hline Gentamicin & $04(45 \%)$ & $05(55 \%)$ \\
\hline Imipenem & $08(89 \%)$ & $01(11 \%)$ \\
\hline Levofloxacin & $06(65 \%)$ & $03(35 \%)$ \\
\hline Meropenem & $07(77 \%)$ & $02(22 \%)$ \\
\hline Cotrimoxazole & $06(67 \%)$ & $03(33 \%)$ \\
\hline
\end{tabular}

Table 6: Antibiotic susceptibility pattern of Klebsiella spp.

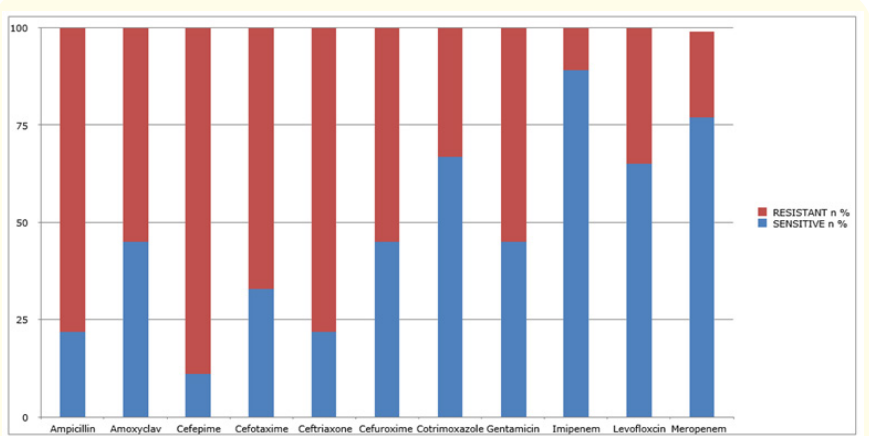

Figure 8: Antibiotic susceptibility pattern of Klebsiella spp.

\begin{tabular}{|l|c|c|}
\hline \multicolumn{1}{|c|}{ Antibiotics } & Sensitive (n \%) & Resistant (n \%) \\
\hline Aztreonam & $08(37 \%)$ & $14(63 \%)$ \\
\hline Ceftazidime & $10(48 \%)$ & $12(51 \%)$ \\
\hline Ciprofloxacin & $17(75 \%)$ & $05(24 \%)$ \\
\hline Levofloxacin & $19(87 \%)$ & $03(12 \%)$ \\
\hline Meropenem & $17(75 \%)$ & $05(25 \%)$ \\
\hline Piperacillin & $17(75 \%)$ & $05(25 \%)$ \\
\hline Piperacillin-tazobactam & $19(87 \%)$ & $03(12 \%)$ \\
\hline Tobramycin & $14(66 \%)$ & $08(33 \%)$ \\
\hline Ticarcillin & $13(59 \%)$ & $09(41 \%)$ \\
\hline
\end{tabular}

Table 7: Antibiotic susceptibility pattern of Pseudomonas spp.

2001 who observed that CONS were most commonly isolated microorganism among BSIs in ICU about 47\% [17]. In a similar study done by Rello., et al. [18] and Valles., et al. [19] CONS accounted for $49.8 \%$ of the isolates. In the present study Gram negative bacilli accounted for $54 \%$ of BSIs in ICU and Pseudomonas aeruginosa accounted for $37 \%$ of these. This is in contrast to study by Cheol- 


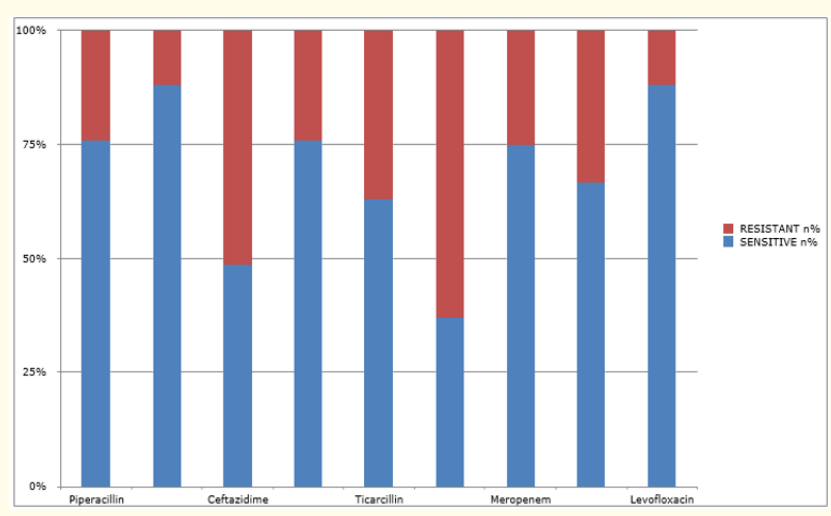

Figure 9: Antibiotic susceptibility pattern of Pseudomonas spp.

\begin{tabular}{|l|c|c|}
\hline \multicolumn{1}{|c|}{ Antibiotics } & Sensitive (n \%) & Resistant (n \%) \\
\hline Ciprofloxacin & $04(50 \%)$ & $04(50 \%)$ \\
\hline Cefoxitin & $03(37 \%)$ & $05(63 \%)$ \\
\hline Clindamycin & $06(75 \%)$ & $02(25 \%)$ \\
\hline Erythromycin & $02(25 \%)$ & $06(75 \%)$ \\
\hline Gentamicin & $02(25 \%)$ & $06(75 \%)$ \\
\hline Levofloxacin & $05(63 \%)$ & $03(37 \%)$ \\
\hline Linezolid & $08(100 \%)$ & 0 \\
\hline Penicillin & $05(63 \%)$ & $03(37 \%)$ \\
\hline Vancomycin & $08(100 \%)(M I C)$ & 0 \\
\hline
\end{tabular}

Table 8: Antibiotic susceptibility pattern of Staphylococcus aureus.

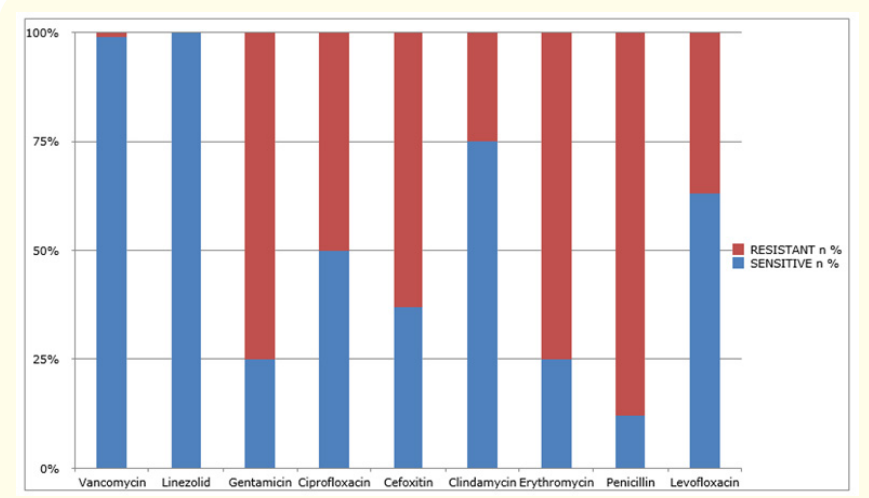

Figure 10: Antibiotic susceptibility pattern of Staphylococcus aureus.

In Kang, Sung-Han Kim., et al. [20], who observed that the most common strains isolated from the bloodstream were Escherichia coli (39.5\%), Klebsiella pneumoniae (20.7\%), Enterobacter species (8.0\%) and P. aeruginosa (7.5\%). From amongst the bacteria isolated in our study, CONS showed $100 \%$ sensitivity to Vancomycin,
$90 \%$ sensitivity to linezolid, $76 \%$ to gentamicin, $54 \%$ sensitivity to levofloxacin. The organisms were found highly resistant to erythromycin (76\%) followed by penicillin (65\%). The second common isolate among GPC was S. aureus which showed $100 \%$ sensitivity to linezolid and vancomycin (100\%), followed by clindamycin (75\%). The organisms were found highly resistant to Penicillin (87\%) followed by erythromycin (75\%) and cefoxitin (62\%). The antimicrobial susceptibility testing revealed that resistance to penicillin was frequent in S. aureus (87\%) and CONS (65\%) this is consistent with the study of I Roy, A Jain, M Kumar, SK Agarwal [12]. Among gram negative organisms Pseudomonas aeruginosa was the most common isolate in which Piperacillin + Tazobactam (87\%) emerged as most sensitive drug followed by piperacillin (75\%), meropenem (75\%) and ciprofloxacin (75\%). Imipenem (92\%) emerged as most sensitive drug against Klebsiella followed by cotrimoxazole (67\%). Strains of Klebsiella showed high resistance for ceftriaxone (78\%), amoxyclav (55\%) followed by Ampicillin (33\%). In our study we found that Citrobacter spp is highly sensitive to imipenem (75\%) and appear resistant to ampicillin (62\%).

\section{Conclusion}

Blood stream infections continue to be a significant source of morbidity among critically ill patients. The results of our study extend knowledge of these infections their causative agents and antibiotic susceptibility. Our findings suggest that, although these infections do not in and of themselves contribute to mortality in the ICU, they may result in increased suffering and costs for patients in the form of prolonged stays and sepsis.

\section{Bibliography}

1. Kuppermann N. "Occult bacteremia in young febrile children". Pediatric Clinics of North America 46.6 (1999): 1073-1110.

2. Bryan C. "Clinical implication if Positive blood culture". Clinical Microbiology Reviews 2.4 (1989): 329-353.

3. Ammerlaan H., et al. "Secular trends in nosocomial bloodstream infections: antibiotic-resistant bacteria increase the total burden of infection". Clinical Infectious Diseases 56.6 (2013): 798-805.

4. Magiorakos AP., et al. "Multidrug-resistant, extensively drugresistant and pandrug-resistant bacteria: an international expert proposal for interim standard definitions for acquired resistance". Clinical Microbiology and Infection 18.3 (2012): 268-281. 
5. Montero JG., et al. "Combatting resistance in intensive care: the multimodal approach of the Spanish ICU "Zero Resistance" program". Critical Care 19 (2015): 114.

6. Cortegiani A., et al. "Use of Cepheid Xpert Carba-R for rapid detection of carbapenemase- producing bacteria in critically ill, abdominal surgical patients: first report of an observational study". Critical Care 19.1 (2015): 108.

7. Timsit JF and Laupland KB. "Update on bloodstream infections in ICUs". Current Opinion in Critical Care 18.5 (2012): 479-486.

8. Carlet J., et al. "Society's failure to protect a precious resource: antibiotics". Lancet 378.9788 (2011): 369-371.

9. Pronovost P., et al. "An intervention to decrease catheter-related bloodstream infections in the ICU". The New England Journal of Medicine 355.26 (2006): 2725-2732.

10. Berenholtz SM., et al. "Eliminating catheter-related bloodstream infections in the intensive care unit". Critical Care Medicine 32.10 (2004): 2014-2020.

11. Fridkin SK., et al. "The role of understaffing in central venous catheter- associated bloodstream infection". Infection Control and Hospital Epidemiology 17.3 (1996): 150-158.

12. Ben-David D., et al. "Outcome of carbapenem resistant Klebsiella pneumoniae bloodstream infections". Clinical Microbiology and Infection 18.1 (2012): 54-60.

13. Panceri M., et al. "An etiology and prognosis of bacteremia in Italy". Epidemiology and Infection 132.4 (2004): 647-654.

14. G Schonheyder H Sorensen. "Source of infection and other factors associated with case fatality in community acquired bacteremia - a Danish population based cohort study from 1992-1997". Clinical Microbiology and Infection 9.8 (2003) :793-802.

15. Ali H., et al. "Etiological agents of bacteremia among newbporns in Hilla city". Medical Journal of Babylon 5.4 (2008): 385-394.

16. Vincenzo Russotto., et al. "Bloodstream infections in intensive care unit patients: distribution and antibiotic resistance of bacteria". Infection and Drug Resistance 8 (2015): 287-296.
17. David K Warren., et al. "Nosocomial Primary Bloodstream Infections in Intensive Care Unit Patients in a Non teaching Community Medical Center: A 21-Month Prospective Study". Clinical Infectious Diseases 33.8 (2001): 1329-1335.

18. Valles J., et al. "Nosocomial bacteremia in critically ill patients: a multicenter study evaluating epidemiology and prognosis". Clinical Infectious Diseases 24 (1997): 387-395.

19. Kang CI., et al. "Bloodstream infections caused by antibioticresistant Gram-negative bacilli: risk factors for mortality and impact of inappropriate initial antimicrobial therapy on outcome". Antimicrobial Agents and Chemotherapy 49.2 (2005): 760-766.

20. I Roy., et al. "Bacteriology of neonatal septicaemia in a tertiary care hospital of northern India". Indian Journal of Medical Microbiology 20.3 (2002): 156-159.

\section{Assets from publication with us}

- Prompt Acknowledgement after receiving the article

- Thorough Double blinded peer review

- Rapid Publication

- Issue of Publication Certificate

- High visibility of your Published work

Website: https://www.actascientific.com/

Submit Article: https://www.actascientific.com/submission.php Email us: editor@actascientific.com

Contact us: +919182824667 